\title{
Discussion on Basic Equations With Source Terms in Compressive and Incompressive Plasmas
}

\author{
Kun-Mu Chen
}

\begin{abstract}
Contribution From the Department of Electrical Engineering, Michigan State University, East Lansing, Mich.
\end{abstract}
(Received August 31, 1964)

\begin{abstract}
It is shown that the same set of basic equations with source terms can and should be applied to both a compressive and an incompressive isotropic plasma. In a compressive plasma, an electric source can excite an electroacoustic wave. In an incompressive plasma, however, this electroacoustic wave reduces to an accumulation of charges shielding the electric source. The physical meaning of the dielectric constant of an incompressive plasma is discussed and the physical model of an incompressive plasma is reexamined.
\end{abstract}

\section{Introduction}

When an electric source is placed in a compressive (hot) plasma, the plasma is generally regarded as an electron fluid with the motion of positive ions neglected. The basic equations for this case are Maxwell equations with source terms and the moment equations which are derived from the Boltzmann equation. When an electric source is immersed in an incompressive (cold) plasma, the plasma is usually considered as a dielectric medium with a dielectric constant of $\epsilon=\epsilon_{0}\left(1-\omega_{\mathrm{p}}{ }^{2} / \omega^{2}\right)$ if no loss is assumed. In this case, Maxwell equations with source terms are adequate to treat the problem. It is usual that two rather different physical models and two different sets of basic equations are adapted for the compressive and the incompressive plasmas. In this paper, it is shown that the same set of basic equations can and should be applied to both kinds of plasmas. The incompressive plasma is just a limiting case of a compressive plasma.

Cohen [1962] and other workers [Hessel and Shmoys, 1962; Chen, 1963; Wait, 1964] have shown that an electric source placed in a compressive plasma can excite an electroacoustic wave in addition to an electromagnetic (EM) wave. In Cohen's analysis, the fields are separated into the EM and the plasma modes under some conditions. In fact, the excitation of an electroacoustic wave in a compressive plasma by an electric source can be derived directly from the basic equations without the modal separation. We also show that the electroacoustic wave excited by an electric source stops propagating and becomes an accumulation of charges surrounding the electric source when the temperature of the plasma approaches zero or the plasma becomes incompressive. This accumulation of charges essentially shields the electric source so that an incompressive plasma behaves as a dielectric medium with $\epsilon=\epsilon_{0}\left(1-\omega_{\mathrm{p}}{ }^{2} / \omega^{2}\right)$ to an electric source. A dielectric model for an incompressive plasma is found to be not entirely satisfactory if an electric source is present.

\section{Basic Equations With Source Terms for a Compressive Plasma}

We assume that an electric source with the current and charge density, $\vec{J}^{s}$ and $\rho^{s}$, is placed in a compressive plasma which is homogeneous and neutral. No static electric or magnetic field is present. If the perturbation of the plasma due to the electric source is small, we can write the basic equations governing this system as follows:

Maxwell equations are

$$
\begin{aligned}
& \nabla \times \vec{E}=-\mu_{0} \frac{\partial \vec{H}}{\partial t} \\
& \nabla \times \vec{H}=\overrightarrow{J^{s}}-e n_{0} \vec{V}+\epsilon_{0} \frac{\partial \vec{E}}{\partial t}
\end{aligned}
$$

and the linearized moment equations are

$$
\begin{gathered}
n_{0} \nabla \cdot \vec{V}+\frac{\partial n_{1}}{\partial t}=0 \\
n_{0} m\left(\frac{\partial \vec{V}}{\partial t}\right)=-n_{0} e \vec{E}-m v_{0}^{2} \nabla n_{1} .
\end{gathered}
$$


The continuity equation which relates $\vec{J}^{s}$ and $\rho^{s}$ is

$$
\nabla \cdot \overrightarrow{J^{s}}+\frac{\partial \rho^{s}}{\partial t}=0 .
$$

In the above equations, $\vec{E}$ and $\vec{H}$ are the electric and magnetic fields, $\vec{V}$ and $v_{0}$ are the mean induced velocity and the rms velocity of electrons, $n_{0}$ and $n_{1}$ are the ambient electron density and the perturbation of the electron density, $m$ and $e$ are the mass and the charge (magnitude) of the electron, and $\epsilon_{0}$ and $\mu_{0}$ are the permittivity and permeability of free space. The rms velocity of electron, $v_{0}$, is expressed as

$$
v_{0}=\sqrt{\frac{3 k T}{m}}
$$

on the assumption of adiabatic pressure variation and one-dimensional compression.

Equations (1) to (5) are the basic equations for a compressive plasma when an electric source is present. Some auxiliary equations can be derived from the basic equations as follows:

Taking the divergence of (2), we have

$$
\nabla \cdot \overrightarrow{J^{s}}-e n_{0} \nabla \cdot \vec{V}+\epsilon_{0} \frac{\partial}{\partial t} \nabla \cdot \vec{E}=0
$$

If (5) and (3) are used to replace $\nabla \cdot \vec{J}^{s}$ and $\nabla \cdot \vec{V}$, (7) becomes

$$
\frac{\partial}{\partial t}\left[\epsilon_{0} \nabla \cdot \vec{E}+e n_{1}-\rho^{s}\right]=0 .
$$

This gives an important equation for $\nabla \cdot \vec{E}$ as

$$
\nabla \cdot \vec{E}=\frac{\rho^{s}}{\epsilon_{0}}-\frac{e n_{1}}{\epsilon_{0}} .
$$

Taking the divergence of (4) and the time derivative of (7), and after $\nabla \cdot \vec{V}$ is eliminated, we have another equation for $\nabla \cdot \vec{E}$ as

$$
\left(\frac{\partial^{2}}{\partial t^{2}}+\omega_{p}^{2}\right) \nabla \cdot \vec{E}=\frac{1}{\epsilon_{0}} \frac{\partial^{2} \rho^{s}}{\partial t^{2}}-\frac{e v_{0}^{2}}{\epsilon_{0}} \nabla^{2} n_{1}
$$

where

$$
\omega_{p}^{2}=\frac{n_{0} e^{2}}{c_{0} m} \text {. }
$$

Since (8) and (9) are derived from the same set of basic equations, they can be combined to yield an equation for $n_{1}$. The substitution of (8) in (9) gives

$$
\left[\nabla^{2}-\frac{1}{v_{0}^{2}} \frac{\partial^{2}}{\partial t^{2}}-\frac{\omega_{p}^{2}}{v_{0}^{2}}\right] n_{1}=-\frac{\omega_{p}^{2}}{e v_{0}^{2}} \rho^{s}
$$

Equation (10) is an inhomogeneous Klein-Gordon equation. This equation directly relates $n_{1}$ to the charge density $\rho^{s}$ of the electric source. When $\rho^{s}$ oscillates with time as $e^{j \omega t},(11)$ becomes

$$
\left[\nabla^{2}+\beta_{p}^{2}\right] n_{1}=-\frac{\omega_{p}^{2}}{e v_{0}^{2}} \rho^{s}
$$

where

$$
\beta_{p}=\frac{1}{v_{0}} \sqrt{\omega^{2}-\omega_{p}^{2}}
$$

Equation (12) is a simple wave equation for $n_{1}$ and implies that $n_{1}$ is excited by $\rho^{s}$ and propagates with a propagation constant of $\beta_{p}$. Since the wave of $n_{1}$ is acoustic type and excited by the electric charge, this wave is called the electroacoustic wave.

\section{Basic Equations With Source Terms for an Incompressive Plasma}

In this section, we aim to show that the same set of basic equations, (1) to (5), still apply in an incompressive plasma and they reduce to those equations used in the dielectric model of the plasma under some conditions.

In an incompressive plasma, the temperature is hypothetically assumed to approach zero. As the result, it is assumed that

$$
v_{0} \rightarrow 0 \text {. }
$$

With (14), the basic equations (1) to (5) are reduced to

$$
\begin{gathered}
\nabla \times \vec{E}=-\mu_{0} \frac{\partial \vec{H}}{\partial t} \\
\nabla \times \vec{H}=\overrightarrow{J^{s}}-e n_{0} \vec{V}+\epsilon_{0} \frac{\partial \vec{E}}{\partial t} \\
n_{0} \nabla \cdot \vec{V}+\frac{\partial n_{1}}{\partial t}=0 \\
n_{0} m\left(\frac{\partial \vec{V}}{\partial t}\right)=-n_{0} e \vec{E} \\
\nabla \cdot \vec{J}^{s}+\frac{\partial \rho^{s}}{\partial t}=0 .
\end{gathered}
$$

These are the basic equations for an incompressive plasma. Some of them are repeated here for the sake of completeness.

The important point in connection with these basic equations is the $\partial n_{1} / \partial t$ term in (17). One may argue that since no electroacoustic wave propagates $n_{1}$ should be zero. This is only true in the source-free region. In case a source is present in the incompressive plasma, $n_{1}$ is not zero at the source point and should not be ignored. In effect, when $v_{0} \rightarrow 0$, (11) gives an equation relating $n_{1}$ to $\rho^{s}$ as

$$
\left[\frac{\partial^{2}}{\partial t^{2}}+\omega_{p}^{2}\right] n_{1}=\frac{\omega_{p}^{2}}{e} \rho^{s} .
$$

Equation (20) implies that $n_{1}$ is not a wave any more; 
$i_{t}$ is rather an accumulation of charges at the source point

When $\rho^{s}$ oscillates with time as $e^{j \omega t}, n_{1}$ is determined from (20) as

$$
n_{1}=-\frac{\omega_{p}^{2}}{\left(\omega^{2}-\omega_{p}^{2}\right) e} \rho^{s} .
$$

The $n_{1} e$ in (21) gives the amount of charges of opposite sign surrounding the electric source $\rho^{s}$. This is the shielding effect of an electric source by the charges in the plasma.

If $(21)$ is substituted in (8), the equation for $\nabla \cdot \vec{E}$ becomes

$$
\nabla \cdot \vec{E}=\frac{\rho^{s}}{\epsilon_{0}\left(1-\omega_{p}^{2} / \omega^{2}\right)} .
$$

Equation (22) implies that the incompressive plasma has a dielectric constant,

$$
\epsilon=\epsilon_{0}\left(1-\omega_{p}^{2} / \omega^{2}\right) .
$$

Physically, it is due to the shielding effect of the electric source by the charges in the plasma which makes the plasma look like a dielectric medium. For this reason, the incompressive plasma behaves as a free-charge medium in the vicinity of an electric source.

If $\vec{V}$ is written as

$$
\vec{V}=j \frac{n_{0} e^{2}}{\omega m} \vec{E}
$$

from (18) for the case of $e^{j \omega t}$ time variation, (16) can be changed to

$$
\nabla \times \vec{H}=\vec{J}^{s}+j \omega \epsilon_{0}\left(1-\omega_{p}^{2} / \omega^{2}\right) \vec{E} .
$$

Equation (25) also implies that the incompressive plasma has an equivalent dielectric constant, $\boldsymbol{\epsilon}=\epsilon_{0}\left(1-\omega_{p}^{2} / \omega^{2}\right)$. However, the physical process involved in achieving this is due to the fact that the induced velocity of electrons is $90^{\circ}$ out of phase of $\vec{E}$ field. This phenomenon is closely related to that in a dielectric medium and, therefore, an incompressive plasma can be considered as a dielectric medium at this point.

By combining (22) and (25) and the physical processes associated with them, one concludes that when an electric source is immersed in an incompressive plasma, a dielectric model for the plasma is acceptable in the source-free region but in the source region a free charge model is more accurate from the physical point of view.

A last remark seems necessary. While a dielectric model for an incompressive plasma for the case of harmonic oscillation is quite adequate, a more accurate model as discussed above and the basic equations (15) to (19) seems more appropriate when a nonsteady electric source is present in the plasma.

\section{References}

Chen, Kun-Mu (1963), Interaction of a radiating source with a plasma, Program Digest, 252-257, Symposium on Antennas and Propagation, Boulder, Colo. 9-11 July 1963 , also appearing in full length in Proc. IEE (London), Oct. 1964, 1668-1678.

Cohen, M. H. (1962), Radiation in a plasma, Phys. Rev. 126 , 389-404.

Hessel, A., and J. Shmoys (1962), Excitation of plasma waves in a homogeneous isotropic plasma by a dipole, Proceedings of the Symposium on Electromagnetics and Fluid Dynamics of Gaseous Plasma, 173-184 (Polytechnic Press, Brooklyn, N.Y.).

Wait, J. R. (1964), Theory of slotted-sphere antenna immersed in a compressive plasma. Part I and II, Radio Sci. J. Res. NBS/USNC-URSI 68D, No. 10, 1127-1136; 1137-1143.

(Paper 69D2-460) 